\title{
Expert system for web quality measurement
}

\author{
Bogdan Walek \\ Institute of Research and Applications of Fuzzy Modeling \\ University of Ostrava \\ Ostrava, Czech Republic \\ bogdan.walek@osu.cz
}

\begin{abstract}
Currently, there are a lot of web pages and web applications which are used for information administration, exchange and publication. However, the quality of the websites is very different. Nowadays there is the user testing approach for web quality measurement which increasing the level of the web quality. In this article, the fuzzy approach and expert system for complex web quality measurement is proposed. The proposed approach is based on evaluating results of user questionnaires and provides a complex evaluation of web application quality. The output of the expert system is the degree of web application quality.
\end{abstract}

Keywords- web quality; measurement; expert system; fuzzy; fuzzy approach; questionnaire; user testing; evaluation

\section{INTRODUCTION}

Various types of web pages and web applications are used for information administration, exchange and publication [1], [2]. There are a few types of information which are published on web pages and applications: texts, images, videos, audio files, animations, etc. Various types of information published on web pages create different impression on users who browse these pages. In addition to this aspect, there are another attributes and properties that affect the behavior of users. Here are a few examples of these attributes [2], [9], [10], [11]:

- clarity of graphical layout

- accessibility of information

- $\quad$ reliability of published content

- meaningful categorization of information

- user friendly navigation and structure

It is relatively difficult to measure the quality of these attributes, but they strongly affect the user's behavior. If one or more of these attributes are weak, the user might have problems with finding relevant information on website and it extends the time which user needs to find what he wants. Eventually, for this reason the user may never return to the website. To verify the accuracy and strength of these important attributes, user testing is performed. The user testing is provided during the design and implementation of the web pages and applications. The main goal of the user testing is to find and fix the critical parts and modules of website which make users confused and nervous. In these parts, users cannot find appropriate information or they must use lengthy and difficult procedures. The output of the user testing procedure is the list of deficiencies and errors which must be fixed or changed. Today, there are a few methods of user testing [3], [4]:

1. Scenario test - users get scenarios with specific instructions or tasks associated with website, users perform the tasks and describe how difficult it was to fulfill them

2. Card sorting - the method is based on selecting appropriate terms associated with website, users write and sort these terms, at the end of this process there is a list of ideas for words and synonyms for navigation labels, links, headlines and search engine optimization

3. Eye tracking - users browse the website with an eye camera (the device that monitors the movement of the eye), and based on collected data, the main and the most monitored parts of web are detected

\section{PROBLEM FORMULATION}

Subsequently, the results of the user testing procedure are processed and the main detected problems are fixed and repaired. But user testing methods test only a few important attributes of website and the complexity of these methods is rather low. It would be better if the user can test all main attributes at one time and refer to the complex output of the website testing. For this reason, it would be appropriate to provide the first user testing during the design and implementation of website, and the second testing after activating the website.

\section{PROBLEM SOLUTION}

Based on these statements, the fuzzy approach with expert system for web quality measurement is proposed. The main goal of the proposed approach is providing the method for complex testing of website quality based on the user's experience and expert's knowledge. The main parts of the fuzzy approach are questionnaires created for users and the expert system which evaluates the results of the questionnaires collected from the users (testers).

The proposed approach is shown in Fig. 1: 


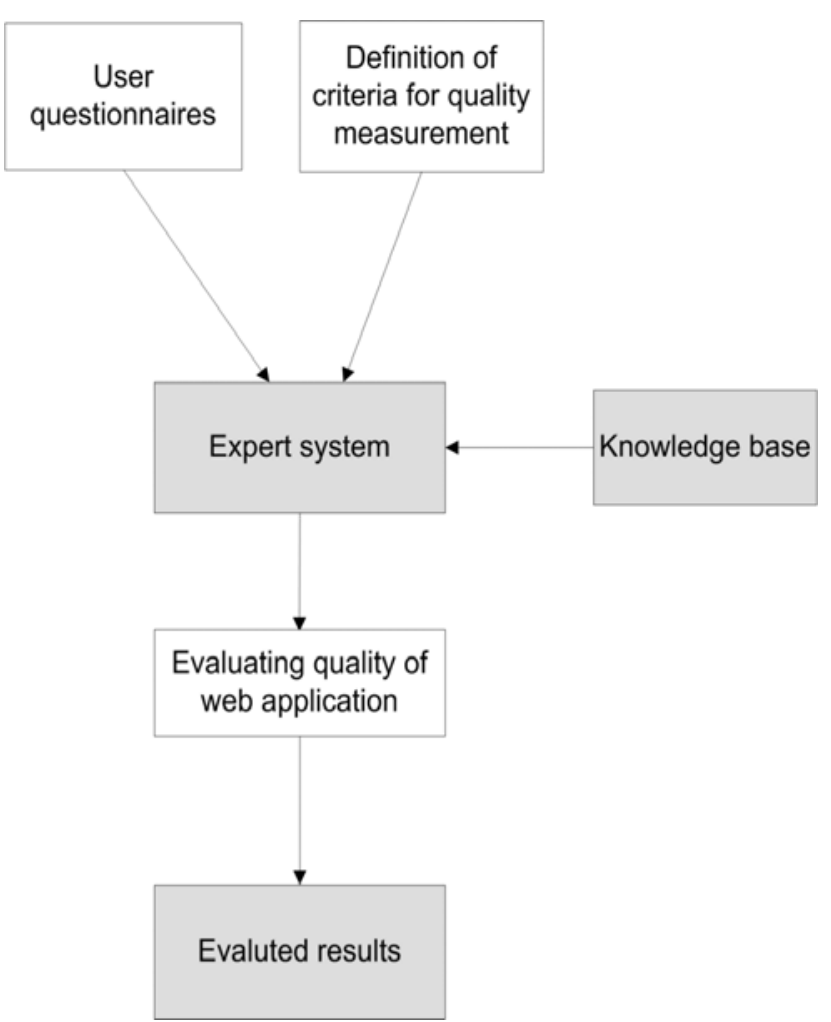

Figure 1. Proposed approach for web quality measurement.

The main steps of the proposed fuzzy approach for web quality measurement are:

1. Definition of criteria for measuring web application quality

2. Creating questionnaire for users

3. Collecting questionnaire results from users

4. Creating expert system

5. Evaluating quality of web application

6 . Visualization of evaluated results

The main step of the proposed approach is to define appropriate criteria for testing the websites and web applications. The criteria should be defined pursuant to main marketing and business targets of the specific website. It is also important to design and fulfill the knowledge base of proposed expert system properly. In specific IF-THEN rules, some criteria may have greater importance than others. The proposed fuzzy approach is based on a general model for decision making process [5]. The general model was also published in [6], [7], [8]. The proposed fuzzy approach will be described in more detail in the following chapter.

\section{FUZZY APROACH AND EXPERT SYSTEM}

\section{A. Definition of criteria for measuring web application quality}

At the beginning, it is very important to define and describe the main criteria for measuring web application quality. As it was mentioned earlier, the criteria depend on the main goals of the web application. For example, intranet web portal is focused more on reliable and current information than on the best graphical layout or accessibility of the website. Here is a list of possible criteria for measuring web application quality:

$$
\begin{array}{ll}
\text { - } & \text { clarity } \\
\text { - } & \text { reliability } \\
\text { - } & \text { accessility } \\
\text { - } & \text { current information } \\
\text { - } & \text { correctness of web structure }
\end{array}
$$

\section{B. Creating questionnaire for users}

In this step, the user questionnaire for users should be created. The user questionnaire will be created based on criteria defined in the previous step. The user will respond to questions which evaluate the web application comprehensively. Each question is associated with specific criteria. The answers are created from linguistic expressions which are for users easy to understand. Here are a few questions of the questionnaire for users:

1. How clear is the main navigation of the web application? (answers: very, medium, little)

2. What is the level of published information? (answers: very high, high, medium, low, very low)

3. What is the level of correctness of web structure (answers: very high, high, medium, low, very low)

\section{Collecting questionnaire results from users}

Subsequently, the answers from the users and their questionnaires are collected and prepared for evaluation by the expert system. In this step, the questionnaires need to be categorized by their importance. For example, questionnaires from elder users may be more important than from young users (because young users have better knowledge of web applications and activities on websites). Example of collected questionnaire results is shown below:

TABLE I. COLLECTED QUESTIONNAIRE RESULTS

\begin{tabular}{|c|c|c|c|}
\hline Users & Question 1 & Question 2 & Question 3 \\
\hline User 1 & very & very high & high \\
\hline User 2 & very & high & medium \\
\hline User 3 & medium & medium & medium \\
\hline
\end{tabular}

\section{Creating expert system}

Then, an expert system with knowledge base will be created. The knowledge base of the expert system consists of a set of IF-THEN rules. IF-THEN rules are created based on criteria for measurement and answers of the questionnaires. The output of the expert system will be the degree of quality of web application:

Here are a few examples of IF-THEN rules: 


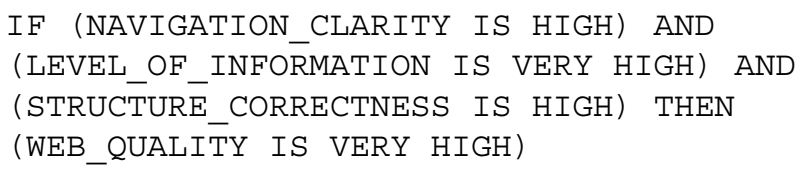

\section{E. Evaluating quality of web application}

In this step, results of the questionnaires will be evaluated by the expert system. Each questionnaire will be evaluated independently. The results of each questionnaire will be processed by the expert system and then, the degree of quality will be associated with each result.

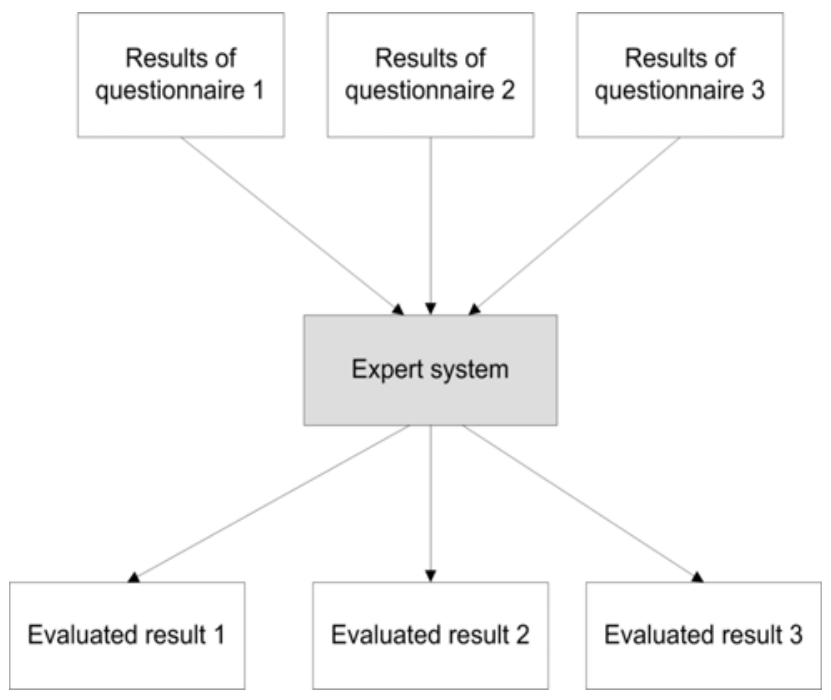

Figure 2. Evaluating results of questionnaires by expert system.

As we can see in Fig. 2, results of all questionnaires are processed by expert system, which evaluates results and associates the degree of quality with each result.

\section{F. Visualization of evaluated results}

Finally, the evaluated results of the web quality measurement will be visualized and shown to the web developer or the project manager. The degree of web quality obtained from evaluated results will be shown using a text and graphical representation. The visualization of the evaluated results is shown in Fig. 3:

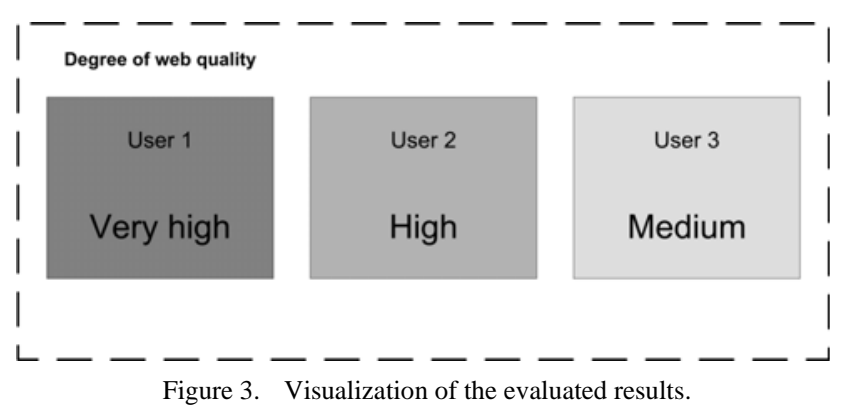

\section{CONCLUSION AND FUTURE WORK}

In this paper, the current state of web quality measurement was analyzed. The current approach for web quality measurement with user testing was identified. Then, the main disadvantages of the current approach were analyzed. The fuzzy approach with the expert system for web quality measurement was proposed. The main steps of the proposed approach were described.

In the future work, attention will be paid to the application of the proposed approach for testing various types of web application and websites. Then, the questionnaire for users will be extended to more complicated questions and answers. Each criterion for measuring web application quality may be supplemented by degree of importance with associated rules in knowledge base of expert system.

\section{REFERENCES}

[1] J. E. Alexander and M. A. Tate, "Web wisdom: how to evaluate and create information quality on the web”, Mahwah, NJ: Erlbaum ,1999.

[2] M. Eppler, and P. Muenzenmayer, "Measuring information quality in the web context: A survey of state-of-the-art instruments and an application methodology”, Proceedings of 7th International Conference on Information Quality, pp.187-196, 2002.

[3] J. Nielsen J. and T. K. Landauer, "A mathematical model of the finding of usability problems”, Proceedings of ACM INTERCHI'93 Conference, Amsterdam , pp. 206-213, 1993.

[4] Nielsen Norman Group, http://www.nngroup.com, 2013.

[5] C. Klimeš, "Model of adaptation under indeterminacy“, Kybernetika, Praque, vol.47, no.3, pp. 355 - 368, Prague 2011.

[6] J. Bartoš, J. Procházka, C. Klimeš, B. Walek and M. Pešl, "Fuzzy reasoning model for decision making under uncertainty”, 16th International Conference on Soft Computing Mendel 2010, Brno, pp. 203-209, 2010.

[7] J. Bartoš, B. Walek, P. Smolka, J. Procházka and C. Klimeš, "Fuzzy modeling tools for information system testing”, 17th International Conference on Soft Computing Mendel 2011, Brno, pp. 154-161, 2011.

[8] B. Walek and C. Klimeš, "Fuzzy tool for conceptual modeling under uncertainty", Fourth International Conference on Machine Vision, 
Proceedings of SPIE vol. 8349, Bellingham, pp. 83492Z-83492Z-5, 2012

[9] M. Eppler, “A Generic Framework for Information Quality in Knowledge-intensive Processes”, Proceedings of the Sixth International Conference on Information Quality, MIT, pp. 329-346, 2001.

[10] K. T. Huang, Y. W. Lee, R.Y. Wang, "Quality Information and Knowledge”, New Jersey: Prentice Hall, 1999.

[11] L. English, "Improving Data Warehouse and Business Information Quality”, Wiley \& Sons: New York, 1999. 